\title{
Higher rate of COVID-19 mortality in patients with type 1 than type 2 diabetes: a nationwide study
}

\author{
Ibrahim Demirci ${ }^{\circledR 1}$, Cem Haymana ${ }^{\circledR 1}$, Ilker Tasci ${ }^{\circledR 2}$, Ilhan Satman ${ }^{\circledR 3,4}$, Aysegul Atmaca ${ }^{(5)}$, \\ Mustafa Sahin ${ }^{(6)}$, Naim Ata ${ }^{\left({ }^{7}\right.}$, Ugur Unluturk ${ }^{\left({ }^{8}\right.}$, Erman Cakal ${ }^{(109}$, Selçuk Dagdelen ${ }^{(18}$, \\ Ibrahim Sahin ${ }^{10}$, Osman Celik ${ }^{11}$, Derun Ertugrul(12, Tevfik Demir ${ }^{(13}$, Rifat Emral ${ }^{\circledR 6}$, \\ Murat Caglayan ${ }^{14}$, Serpil Salman ${ }^{15}$, Suayip Birinci ${ }^{16}$, Alper Sonmez ${ }^{17}$
}

${ }^{1}$ University of Health Sciences, Gulhane Training and Research Hospital, Department of Internal Medicine, Division of Endocrinology and Metabolism, Ankara, Turkey

${ }^{2}$ University of Health Sciences, Gulhane Faculty of Medicine and Gulhane Training and Research Hospital, Department of Internal Medicine, Ankara, Turkey

${ }^{3}$ Istanbul University, Faculty of Medicine, Department of Internal Medicine, Division of Endocrinology and Metabolism, Istanbul, Turkey ${ }^{4}$ The Health Institutes of Turkey, Institute of Public Health and Chronic Diseases, Istanbul, Turkey

${ }^{5}$ Ondokuz Mayis University, Faculty of Medicine, Department of Internal Medicine, Division of Endocrinology and Metabolism, Samsun, Turkey

${ }^{6}$ Ankara University, Faculty of Medicine, Department of Internal Medicine, Division of Endocrinology and Metabolism, Ankara,

Turkey

${ }^{7}$ Ministry of Health, Department of Strategy Development, Ankara, Turkey

${ }^{8}$ Hacettepe University, Faculty of Medicine, Department of Internal Medicine, Division of Endocrinology and Metabolism, Ankara, Turkey ${ }^{9}$ University of Health Sciences, Faculty of Medicine, Diskapi Yildirim Beyazit Training and Research Hospital, Department of Internal Medicine, Division of Endocrinology and Metabolism, Ankara, Turkey

${ }^{10}$ Inonu University, Faculty of Medicine, Department of Internal Medicine, Division of Endocrinology and Metabolism, Malatya, Turkey

${ }^{11}$ Public Hospitals General Directorate, Republic of Turkey, Ministry of Health, Ankara, Turkey

${ }^{12}$ University of Health Sciences, Faculty of Medicine, Kecioren Training and Research Hospital, Department of Internal Medicine,

Division of Endocrinology and Metabolism, Ankara, Turkey

${ }^{13}$ Dokuz Eylul University, Faculty of Medicine, Department of Internal Medicine, Division of Endocrinology and Metabolism, Ankara, Turkey

${ }^{14}$ Ankara Provincial Health Directorate, Ankara, Turkey

${ }^{15}$ Medica Clinic, Endocrinology and Metabolism, Istanbul, Turkey

${ }^{16}$ Deputy Minister of Health, Ministry of Health, Ankara, Turkey

${ }^{17}$ University of Health Sciences, Gulhane Faculty of Medicine and Training and Research Hospital, Department of Internal Medicine, Division of Endocrinology and Metabolism, Ankara, Turkey

\begin{abstract}
Introduction: COVID-19 disease has a worse prognosis in patients with diabetes, but comparative data about the course of COVID-19 in patients with type 1 (T1DM) and type 2 diabetes (T2DM) are lacking. The purpose of this study was to find out the relative clinical severity and mortality of COVID-19 patients with T1DM and T2DM.

Material and methods: A nationwide retrospective cohort of patients with confirmed (PCR positive) COVID-19 infection ( $\mathrm{n}=149,671)$ was investigated. After exclusion of individuals with unspecified diabetes status, the adverse outcomes between patients with T1DM $(\mathrm{n}=163), \mathrm{T} 2 \mathrm{DM}(\mathrm{n}=33,478)$ and those without diabetes $(\mathrm{n}=115,108)$ were compared by using the propensity score matching method. The outcomes were hospitalization, the composite of intensive care unit (ICU) admission and/or mechanical ventilation, and mortality. Results: The patients with T1DM had higher mortality than the age- and gender-matched patients with T2DM $(\mathrm{n}=489)$ and those without diabetes $(\mathrm{n}=489)(\mathrm{p}<0.001)$. After further adjustment for the $\mathrm{HbA}_{1 \prime^{\prime}}$ and microvascular and macrovascular complications, the odds of mortality (OR: 3.35, 95\% CI: 1.41-7.96, p = 0.006) and ICU admission and/or mechanical ventilation (OR: 2.95, 95\% CI: 1.28-6.77, p = 0.011) were significantly higher in patients with T1DM compared to those with T2DM. Older age (OR: 1.06, 95\% CI: 1.01-1.12, p = 0.028) and lymphopaenia (OR: 5.13, 95\% CI: 1.04-25.5, $\mathrm{p}=0.045$ ) were independently associated with mortality in patients with T1DM.

Conclusions: Patients with T1DM had worse prognosis of COVID-19 compared to T2DM patients or those without diabetes. These cases should be cared for diligently until more data become available about the causes of increased COVID-19 mortality in T1DM. (Endokrynol Pol 2022; 73 (1): 87-95)
\end{abstract}

Key words: COVID-19; coronavirus; type 1 diabetes; mortality; diabetes mellitus; T1DM; Turkey 


\section{Introduction}

Patients with type 1 diabetes mellitus (T1DM) and type 2 diabetes mellitus (T2DM) are at increased risk of lower respiratory tract, urinary system, and skin and mucous membrane infections.[1] People with diabetes are also at increased risk of complicated influenza A (H1N1) infections, with no difference in adverse outcomes between the 2 types of diabetes [2]. As with other infectious aetiologies, certain populations, such as men, the elderly, or those with comorbid diseases, including diabetes mellitus, are more vulnerable to an unfavourable outcomes of new coronavirus disease 2019 (COVID-19) [3-7]. The frequency of T2DM among confirmed COVID-19 patients varies between 10.1\% and $68.3 \%$ in different studies [8-10], and the mortality rate ranges between $8 \%$ and $60 \%$ [11-14].

Little is known about the course of COVID-19 infection in patients with T1DM rather than T2DM [15-17]. So far, only a few studies have reported the prevalence of T1DM in patients with COVID-19 infection, at between $0.6 \%$ and $2.1 \%$, and the mortality rates range from $3 \%$ to $9 \%$. However, these studies were too small to identify the characteristics of this vulnerable population [16, 18-20]. Moreover, potential causes of severe outcomes of COVID-19 have not been sufficiently elucidated in different settings [18-20].

This study aimed to investigate the rates of mortality, hospitalization, and admission to intensive care units (ICU) due to COVID-19 in patients with T1DM in comparison to those with T2DM and no diabetes. Moreover, we addressed the potential factors associated with poor outcomes in T1DM.

\section{Material and methods}

\section{Study design and participants}

This multi-centre retrospective cohort study was carried out using the National Electronic Database of the Turkish Ministry of Health. We identified 149,671 adult patients with a confirmed diagnosis of COVID-19 (PCR positive) between 11 March through 30 May 2020 in the database. After exclusion of individuals with unspecified diabetes status $(\mathrm{n}=922)$, we classified the remaining sample as T1DM $(n=163)$, T2DM $(n=33478)$, or no diabetes $(n=115108)$. To explore the relative risk of adverse outcomes in the T1DM group (see below), age- and gender-matched comparator groups were formed using the propensity score matching (PSM) method in the $\mathrm{T} 2 \mathrm{DM}$ and no diabetes patient datasets. To increase the precision and performance of the PSM procedure, we repeated the matching by propensity scores three times in the T2DM and non-diabetic patients (Fig. 1). After the initial analyses, the PSM groups were pooled, totalling a final sample of 1141 subjects.

The design and procedures in the study are in accordance with the declaration of Helsinki and the study protocol was approved

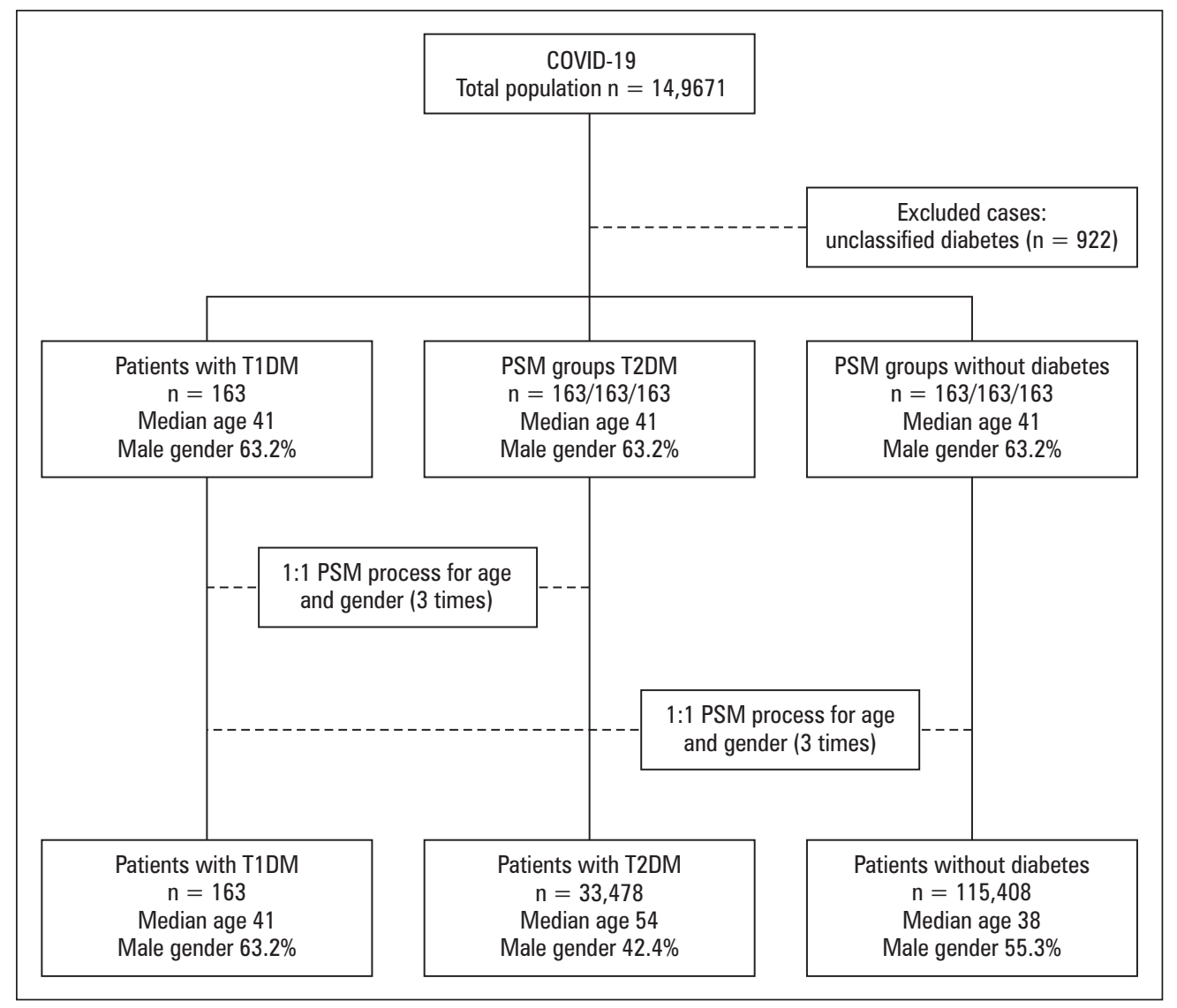

Figure 1. Study inclusion flow chart. T1DM - type 1 diabetes mellitus; T2DM - type 2 diabetes mellitus; PSM - propensity score matching 
by the Ministry of Health Ethical Board (IRB no. 95741342020:186404/28.10.2020).

\section{Data collection}

Sociodemographic data including age, gender, education, smoking body mass index (BMI), comorbid diseases, and medications were recorded. The laboratory parameters obtained from the national database were blood glucose, glycosylated haemoglobin $\mathrm{A} 1 \mathrm{c}\left(\mathrm{HbA}_{1}\right.$ - for patients with diabetes), low-density lipoprotein cholestero (LDL-C), creatinine, aspartate transaminase (AST), alanine transaminase (ALT), C-reactive protein (CRP), lymphocyte count, lactate dehydrogenase (LDH), and ferritin. The tests were performed in hospital laboratories certified by the Turkish Ministry of Health. Chest computerized tomography (CT) reports were available in the national database as positive or negative for COVID-19.

\section{Definitions}

Patients with T1DM were first identified by ICD-10 codes. Each diagnosis of T1DM was further verified within the health insurance database through trace records of the patients. T2DM was defined as previously described [7] using the ICD-10 codes, or having any $\mathrm{HbA}_{1 \mathrm{c}} \geq 6.5 \%$, or monthly refill of antidiabetic medications following the diagnosis of T2DM. Undetermined records of diabetes type were considered "unclassified"; therefore, these patients were excluded from the study.

Smoking was defined as currently smoking at the time of the COVID-19 diagnosis. Higher education was described as the attained level of education for more than eight years. BMI was calculated as the ratio of weight to the square of height $\left(\mathrm{kg} / \mathrm{m}^{2}\right)$. Hypertension, dyslipidaemia, chronic obstructive pulmonary disease (COPD), asthma, heart failure, coronary artery disease, peripheral artery disease, and cerebrovascular disease were identified using the ICD-10 codes. The composite of coronary artery disease, peripheral artery disease, and cerebrovascular disease was recorded as cardiovascular disease (CVD). Obesity was defined as BMI $\geq 30 \mathrm{~kg} / \mathrm{m}^{2}$. Chronic kidney disease was specified as estimated glomerular filtration rate (eGFR) $<60 \mathrm{~mL} / \mathrm{min} / 1.73 \mathrm{~m}^{2}$ using the CKD-EPI equation [21]. Renin-angiotensin system (RAS) medication use was composed of receiving any angiotensin-converting enzyme inhibitors or angiotensin receptor blockers with/without their combination forms

\section{Outcomes}

The primary outcome was mortality due to COVID-19 in patients with T1DM, compared with patients with T2DM and patients without diabetes. The secondary outcomes were hospitalization and the composite of ICU admission and/or mechanical ventilation.

\section{Statistical analyses}

Numerical data were expressed as median (interquartile range-IQR) and categorical variables as count (n) and percentage (\%). Normality of distribution was assessed using the Kolmogorov-Smirnov test. Differences between groups were assessed using the chi-square test for categorical variables and Student's t-test or the Mann-Whitney $\mathrm{U}$ test, as appropriate.

Confirmed T1DM patients $(n=163)$ in the full dataset were matched using the propensity score on a scale of 1:1 by age and gender to patients with T2DM and patients without diabetes. Univariate analyses were performed to evaluate the potential variables associated with mortality, hospitalization, and ICU admission/mechanical ventilation in patients with T1DM and presented as odds ratio (OR) and its 95\% confidence interval (CI). Multivariable logistic regression analysis was used to study the independent predictors of the three outcomes. Variables with significant univariate association with the outcomes and variables, which could be potential predictors despite the lack of significant univariate association, were included in a multivariate model. The Hosmer-Lemeshow test and likelihood ratio test were used to assess final model fitting. Statistical significance was defined as two-sided $p$ values $\leq 0.05$. Kaplan-Meier survival curves were plotted to visualize the difference between 30-day mortality rates of T1DM and PSM control groups. All data were analysed using SPSS Statistics for Windows version 25.0 (SPSS Inc. 111 Chicago, IL).

\section{Results}

\section{Basic characteristics}

Demographic and clinical variables in the overall, unmatched sample are shown in Table 1. Of the total sample of 149,671 patients with confirmed COVID-19 disease, $0.1 \%(\mathrm{n}=163)$ had T1DM, 22.4\% had T2DM ( $\mathrm{n}=33,478)$, and $76.9 \%$ had no diabetes $(\mathrm{n}=115,108)$. The median (IQR) age of patients with T1DM was lower than that of patients with T2DM but higher than that of patients without diabetes ( $p<0.05$ for all). There was a male predominance among patients with T1DM $(63.2 \%)$ and non-diabetics (55.3\%), whereas fewer patients were male among patients with T2DM (42.4\%) (Fig. 1 and Tab. 1). The group with T1DM had more chronic kidney disease and coronary heart disease compared to both the T2DM and no-diabetes groups; a higher rate of microvascular and macrovascular complications than patients with T2DM; and a higher rate of hypertension, dyslipidaemia, and asthma/COPD than the no-diabetes groups ( $p<0.05$ for all) (Tab. 1). The blood glucose level at admission, the proportion of above normal LDH and ferritin, and the proportion of lymphopaenia were also higher in the group with T1DM compared to the T2DM and no-diabetes groups $(\mathrm{p}<0.05)$ (Tab. 1). The proportion of patients using antihyperglycaemic drugs was similar in the groups with T1DM and T2DM, except for insulin therapy. The use of RAS blockers, statins, and acetyl salicylic acid was higher in both diabetes groups than in the no-diabetes group.

After matching the T1DM group $(n=163)$ with three different groups of patients with T2DM and patients without diabetes, the final sample consisted of 1141 individuals, of whom $14.2 \%$ had T1DM, $42.9 \%$ had T2DM ( $\mathrm{n}=489,163$ subjects in each of three PSM groups), and $42.9 \%$ had no diabetes ( $\mathrm{n}=489,163$ subjects in each of 3 PSM groups). The median (IQR) age of the sample was 41 (36) years, and $63.2 \%$ of patients were male (Fig. 1 and Tab. 2). The comparison of demographic and clinical variables after matching is shown in Table 2. The prevalence of hypertension, heart failure, chronic kidney disease, and coronary artery disease was higher when compared to patients with T2DM and those without diabetes $(\mathrm{p}<0.05$ for all). The microvascular and macrovascular complications were more common in patients with T1DM when compared to those with T2DM, and the rates of dyslipidaemia and asthma/COPD were higher than in the no-diabetes group ( $p<0.001$ for all). Increased blood glucose level on admission and lymphopaenia 
Table 1. Basic characteristics and comparison of clinical and demographic parameters of COVID-19 patients with type 1 diabetes mellitus (T1DM), type 2 diabetes mellitus (T2DM), and no-diabetes patients (crude analysis, before PSM)

\begin{tabular}{|c|c|c|c|c|c|}
\hline & $\begin{array}{c}\text { T1DM } \\
(n=163)\end{array}$ & $\begin{array}{c}\text { T2DM } \\
(\mathrm{n}=33,478)\end{array}$ & $\begin{array}{l}\text { No-diabetes } \\
(\mathrm{n}=115,108)\end{array}$ & p1 & p2 \\
\hline Age, years, median (IQR) & $41(36)$ & $54(81)$ & $38(21)$ & $<0.001$ & $<0.001$ \\
\hline Gender, male, $\mathrm{n}(\%)$ & $103(63.2)$ & $14209(42.4)$ & $63703(55.3)$ & $<0.001$ & 0.044 \\
\hline Smoking (current smoker $-n, \%$ ) & $29(25.7)$ & $3612(16.2)$ & $18914(22.3)$ & 0.006 & 0.397 \\
\hline \multicolumn{6}{|l|}{ Follow-up centre, n (\%) } \\
\hline Public hospitals & $118(72.4)$ & $25216(75.3)$ & $92361(80.3)$ & & \\
\hline University hospitals & $17(10.4)$ & $2596(7.8)$ & $6386(5.5)$ & & \\
\hline Private centres & $28(17.2)$ & $5664(16.9)$ & $16342(14.2)$ & 0.431 & 0.010 \\
\hline Education ( 9 years and over $-n, \%$ ) & $10(43.5)$ & $1309(27.9)$ & $6642(39.4)$ & 0.098 & 0.690 \\
\hline \multicolumn{6}{|l|}{ Comorbid conditions } \\
\hline Hypertension, n (\%) & $110(67.5)$ & $22897(68.4)$ & $28497(24.8)$ & 0.803 & $<0.001$ \\
\hline Dyslipidaemia, n (\%) & $80(49.1)$ & $14923(44.6)$ & $8371(7.3)$ & 0.248 & $<0.001$ \\
\hline Obesity, n (\%) & $5(18.5)$ & $2112(49.5)$ & $2136(21.0)$ & 0.001 & 0.755 \\
\hline Asthma/COPD, n (\%) & $57(35.0$ & $11112(33.2)$ & $18222(15.8)$ & 0.631 & $<0.001$ \\
\hline Chronic kidney disease, n (\%) & $43(54.4)$ & $2187(18.8)$ & $1605(7.7)$ & $<0.001$ & $<0.001$ \\
\hline Coronary artery disease (CAD), $\mathrm{n}(\%)$ & 65 (39.9) & $10778(32.2)$ & $9488(8.2)$ & 0.036 & $<0.001$ \\
\hline Cancer, n (\%) & $8(4.9)$ & $2402(7.2)$ & $2954(2.6)$ & 0.263 & 0.059 \\
\hline Microvascular complications, n (\%) & $77(47.2)$ & $6120(18.3)$ & NA & $<0.001$ & NA \\
\hline Macrovascular complications, $\mathrm{n}(\%)$ & $73(44.8)$ & $11864(35.4)$ & NA & 0.013 & NA \\
\hline \multicolumn{6}{|l|}{ Laboratory values } \\
\hline CT findings of COVID-19 & $60(39.0)$ & $10900(34.5)$ & $22281(21.1)$ & 0.251 & $<0.001$ \\
\hline Glucose [mg/dL] median (IQR) & $188(124)$ & $127(78)$ & $103(188)$ & $<0.001$ & $<0.001$ \\
\hline $\mathrm{HbA}_{1 \mathrm{c}}(\%)$, median (IQR) & $8.5(3.1)$ & $7(2.4)$ & NA & $<0.001$ & NA \\
\hline $\mathrm{HbA}_{1 \mathrm{c}}[\mathrm{mmol} / \mathrm{mol}]$, median (IQR) & $69.4(33.6)$ & $53(26.2)$ & NA & $<0.001$ & NA \\
\hline LDL-C [mg/dL], median (IOR) & $117(71)$ & $116(53)$ & $112(52)$ & 0.566 & 0.871 \\
\hline eGFR [mL/min/1.73 m²], median (IOR) & $58(94)$ & $96.4(46)$ & $108(43)$ & $<0.001$ & $<0.001$ \\
\hline AST > ULN, n (\%) & $8(20)$ & $1061(21.1)$ & $1685(17.0)$ & 0.866 & 0.611 \\
\hline ALT > ULN, n (\%) & $2(4.5)$ & $1048(20.6)$ & $1947(19.6)$ & 0.009 & 0.012 \\
\hline CRP > ULN, n (\%) & $52(76.5)$ & $6460(70.0)$ & $10638(56.9)$ & 0.244 & 0.001 \\
\hline Lactate dehydrogenase > ULN, $\mathrm{n}(\%)$ & $20(62.5)$ & $2348(44.2)$ & $3019(33.4)$ & 0.038 & 0.001 \\
\hline Ferritin $>100 \mathrm{ng} / \mathrm{mL}, \mathrm{n}(\%)$ & $36(85.7)$ & $3016(60.7)$ & $3798(49.6)$ & 0.001 & $<0.001$ \\
\hline Lymphopenia, Lym\# < 1000, n (\%) & $31(32.0)$ & $3810(19.9)$ & $8704(15.7)$ & 0.003 & $<0.001$ \\
\hline \multicolumn{6}{|l|}{ Treatments } \\
\hline RAS blocker, $\mathrm{n}(\%)$ & $78(47.9)$ & $15746(47.0)$ & $13889(12.1)$ & 0.834 & $<0.001$ \\
\hline Insulin, n (\%) & $163(100)$ & $7705(23.0)$ & 0 & $<0.001$ & NA \\
\hline Statin, n (\%) & $51(31.3)$ & $8648(25.8)$ & $3047(2.6)$ & 0.112 & $<0.001$ \\
\hline Acetylsalicylic acid, $\mathrm{n}(\%)$ & $58(35.6)$ & $10219(30.5)$ & $8721(7.6)$ & 0.162 & $<0.001$ \\
\hline \multicolumn{6}{|l|}{ Outcomes } \\
\hline Hospitalization, n (\%) & $99(60.7)$ & $18621(55.6)$ & $44648(38.8)$ & 0.190 & $<0.001$ \\
\hline ICU admission and intubation, $\mathrm{n}(\%)$ & $31(31.6)$ & $3832(20.6)$ & $4371(9.8)$ & 0.007 & $<0.001$ \\
\hline Mortality, n (\%) & $26(16.0)$ & $2565(7.7)$ & $2095(1.8)$ & $<0.001$ & $<0.001$ \\
\hline
\end{tabular}

p1: T1DM vs. T2DM; p2: T1DM vs. no diabetes; COPD — chronic obstructive pulmonary disease; CT — computerized tomography; HbA $A_{1 \mathrm{c}}$ - glycosylated haemoglobin $A_{1} ;$ LDL-C — low-density lipoprotein cholesterol; eGFR — estimated glomerular filtration rate; AST — aspartate aminotransferase; ALT — alanine aminotransferase; CRP — C-reactive protein; ULN — upper limit of normal; RAS — renin-angiotensin-aldosterone system; PSM — prospensity score matching; ICU — intensive care unit 
Table 2. Comparison of demographic and clinical parameters among COVID-19 patients with type 1 (T1DM), type 2 (T2DM) diabetes mellitus, and no-diabetes patients

\begin{tabular}{|c|c|c|c|c|c|}
\hline & $\begin{array}{c}\text { T1DM } \\
n=163\end{array}$ & $\begin{array}{c}\text { T2DM } \\
\mathrm{n}=489 \\
\text { (pooled PSM) }\end{array}$ & $\begin{array}{c}\text { No-diabetes } \\
n=489 \\
\text { (pooled PSM) }\end{array}$ & p1 & p2 \\
\hline Age [years], median (IOR) & $41(36)$ & $41(36)$ & $41(36)$ & 1.000 & 1.000 \\
\hline Gender, male, $\mathrm{n}(\%)$ & $103(63.2)$ & $309(63.2)$ & $309(63.2)$ & 1.000 & 1.000 \\
\hline Smoking (current smoker $-\mathrm{n}, \%$ ) & $29(25.7)$ & $71(20.1)$ & $76(21.5)$ & 0.236 & 0.363 \\
\hline \multicolumn{6}{|l|}{ Follow-up centre, $n(\%)$} \\
\hline Public hospitals & $118(72.4)$ & $367(75.1)$ & $406(83.0)$ & & \\
\hline University hospitals & $17(10.4)$ & $33(6.7)$ & $21(4.3)$ & 0.309 & 0.003 \\
\hline Private centres & $28(17.2)$ & $89(18.2)$ & $62(12.7)$ & & \\
\hline Education ( 9 years and over $-n, \%)$ & $10(43.5)$ & $24(31.2)$ & $12(20.7)$ & 0.199 & 0.038 \\
\hline \multicolumn{6}{|l|}{ Comorbid conditions } \\
\hline Hypertension, $n(\%)$ & $110(67.5)$ & $286(58.5)$ & $170(34.8)$ & 0.025 & $<0.001$ \\
\hline Dyslipidaemia, n (\%) & $80(49.1)$ & $222(45.4)$ & $52(10.6)$ & 0.234 & $<0.001$ \\
\hline Obesity, n (\%) & $5(18.5)$ & $31(43.7)$ & $11(30.6)$ & 0.017 & 0.383 \\
\hline Asthma/COPD, n (\%) & $57(35.0)$ & $140(28.6)$ & $88(18)$ & 0.140 & $<0.001$ \\
\hline Hearth failure, n (\%) & $38(23.3)$ & $46(9.4)$ & $16(3.3)$ & $<0.001$ & $<0.001$ \\
\hline Chronic kidney disease, $\mathrm{n}(\%)$ & $43(54.4)$ & $40(22.0)$ & $14(13.1)$ & $<0.001$ & $<0.001$ \\
\hline Coronary artery disease (CAD), $\mathrm{n}(\%)$ & $65(39.9)$ & $135(27.6)$ & $79(16.2)$ & 0.003 & $<0.001$ \\
\hline Microvascular complications, $\mathrm{n}(\%)$ & $77(47.2)$ & $90(18.4)$ & NA & $<0.001$ & NA \\
\hline Macrovascular complications, n (\%) & $73(44.8)$ & $151(30.9)$ & NA & 0.001 & NA \\
\hline Cancer, n (\%) & $8(4.9)$ & $31(6.3)$ & $16(3.3)$ & 0.505 & 0.337 \\
\hline \multicolumn{6}{|l|}{ Laboratory values } \\
\hline CT findings of COVID-19 & $60(39.0)$ & $153(33.5)$ & $110(24.2)$ & 0.217 & $<0.001$ \\
\hline Glucose [mg/dL], median (IQR) & $188.5(124)$ & $139(62)$ & $107(26)$ & 0.001 & $<0.001$ \\
\hline $\mathrm{HbA}_{1 \mathrm{c}}(\%)$, median (IQR) & $8.5(3.1)$ & $6.6(2.5)$ & NA & $<0.001$ & NA \\
\hline $\mathrm{HbA}_{1 \mathrm{c}}[\mathrm{mmol} / \mathrm{mol}]$, median (IOR) & $69.4(33.6)$ & $48.2(27.3)$ & NA & $<0.001$ & NA \\
\hline LDL-C [mg/dL], median (IQR) & $117(72)$ & $109(46)$ & $110(58)$ & 0.694 & 0.664 \\
\hline eGFR $\left[\mathrm{mL} / \mathrm{min} / 1.73 \mathrm{~m}^{2}\right]$, median (IOR) & $58(94)$ & $99(59)$ & $98(49)$ & $<0.001$ & $<0.001$ \\
\hline AST > ULN, n (\%) & $8(20)$ & $15(19.2)$ & $8(14.8)$ & 0.920 & 0.584 \\
\hline ALT > ULN, n (\%) & $2(4.5)$ & $22(27.8)$ & $7(13.7)$ & 0.002 & 0.170 \\
\hline CRP > ULN, n (\%) & $52(76.5)$ & $96(75.0)$ & $48(54.5)$ & 0.820 & 0.005 \\
\hline Lactate dehydrogenase > ULN, n (\%) & $20(62.5)$ & $41(51.9)$ & $22(43.1)$ & 0.309 & 0.086 \\
\hline Ferritin > $100 \mathrm{ng} / \mathrm{mL}, \mathrm{n}(\%)$ & $36(85.7)$ & $47(58.8)$ & $31(67.4)$ & 0.002 & 0.050 \\
\hline Lymphopaenia, Lym\# < 1000, n (\%) & $31(32.0)$ & $53(20.1)$ & $44(16.5)$ & 0.018 & 0.001 \\
\hline \multicolumn{6}{|l|}{ Treatments } \\
\hline RAS blocker, n (\%) & $78(47.9)$ & $192(39.3)$ & $112(22.9)$ & 0.054 & $<0.001$ \\
\hline Insulin, n (\%) & $163(100)$ & $152(31.1)$ & 0 & $<0.001$ & NA \\
\hline Statin, n (\%) & $51(31.3)$ & $127(26.0)$ & $30(6.1)$ & 0.187 & $<0.001$ \\
\hline Acetylsalicylic acid, n (\%) & $58(35.6)$ & $154(31.5)$ & $75(15.3)$ & 0.334 & $<0.001$ \\
\hline \multicolumn{6}{|l|}{ Outcomes } \\
\hline Hospitalization, n (\%) & $99(60.7)$ & $263(53.8)$ & $230(47.0)$ & 0.122 & 0.002 \\
\hline ICU admission and intubation, $\mathrm{n}(\%)$ & $31(31.6)$ & $60(22.8)$ & $25(10.9)$ & 0.086 & $<0.001$ \\
\hline Mortality, n (\%) & $26(16.0)$ & $34(7.0)$ & $14(2.9)$ & 0.001 & $<0.001$ \\
\hline
\end{tabular}

p1: T1DM vs. T2DM; p2: T1DM vs. no diabetes; COPD — chronic obstructive pulmonary disease; CT — computerized tomography; $\mathrm{HbA}_{1 \mathrm{c}}$ - glycosylated haemoglobin $A_{10} ;$ LDL-C — low-density lipoprotein cholesterol; eGFR — estimated glomerular filtration rate; AST — aspartate aminotransferase; ALT — alanine aminotransferase; CRP — C-reactive protein; ULN — upper limit of normal; RAS — renin-angiotensin-aldosterone system; ICU — intensive care unit 
were also more prevalent in the group with T1DM than among patients with T2DM and those without diabetes $(\mathrm{p}<0.05)$ (Tab. 2). Similar to the crude comparisons, the proportion of patients using antihyperglycaemic medications, except for insulin, therapy was similar in the groups with T1DM and T2DM after matching. The use of RAS blockers, statins, and acetyl salicylic acid use was higher in both diabetes groups compared with the no-diabetes group in matched analysis.

\section{Outcome analysis}

A comparison of study outcomes between unmatched groups showed a significantly higher rate of mortality, hospitalization, and the composite of ICU admission and/or mechanical ventilation in patients with T1DM compared to patients without diabetes ( $p<0.001$ for all) (Tab. 1). The rates of mortality and ICU admission and/or mechanical ventilation were also higher than in the T2DM group ( $\mathrm{p}<0.05)$.

Considering the pooled PSM groups, there was a greater risk of mortality in the T1DM group than in the T2DM groups $(\mathrm{p}<0.001)$. No significant difference was observed between the T1DM and T2DM groups regarding hospitalization or ICU admission and/or mechanical ventilation. On the other hand, the risk of mortality, hospitalization, and ICU admission and/or mechanical ventilation in the group with T1DM was higher in comparison to the no-diabetes groups ( $p<0.01$ for all) (Tab. 2). The comparison of outcomes across each PSM group is given in Supplementary File - Table 1.

After further adjustment for the $\mathrm{HbA}_{1 c^{\prime}}$ microvascular, and macrovascular complications, the odds of mortality (OR: 3.35, 95\% CI: 1.41-7.96, p = 0.006) and ICU admission and/or mechanical ventilation (OR: 2.95, 95\% CI: 1.28-6.77, p = 0.011) were significantly higher in patients with T1DM compared to the group with T2DM (Fig. 2). The odds of mortality (OR: 6.44, 95\% CI: 3.27-12.67, $\mathrm{p}<0.001$ ), hospitalization (OR: 1.74, 95\% CI: 1.21-2.50, $\mathrm{p}=0.003$ ), and ICU admission and/or mechanical ventilation (OR: 3.78, 95\% CI: 2.08-6.84, $\mathrm{p}<0.001$ ) were also significantly higher in patients with T1DM compared to the no-diabetes group.

\section{Predictors of outcomes}

Multivariate logistic regression analyses showed that older age (OR: 1.06, 95\% CI: 1.01-1.12, $\mathrm{p}=0.028$ ) and lymphopaenia (OR: 5.13, 95\% CI: 1.04-25.5, p = 0.045) were independently associated with mortality in patients with T1DM. Chest CT findings of COVID-19 (OR: $4.41,95 \%$ CI: 1.12-17.3, $\mathrm{p}=0.033$ ) were associated with an increased risk of hospitalization, while older age (OR: 1.05, 95\% CI: 1.01-1.10, p = 0.016) was associated with a higher risk of ICU admission (Supplementary File - Tab. S2).

\section{The survival curves}

The Kaplan-Meier curves showing the cumulative survival rates of 30-day mortality are displayed for the crude analysis in Figure $3 \mathrm{~A}$ and matched analysis in Figure 3B. Both analyses yielded lower survival rates for patients with T1DM (logrank test $\mathrm{p}<0.001$ ).

\section{Discussion}

The results of this nationwide retrospective observational cohort study showed a significantly higher risk of hospitalization, ICU admission/intubation, and mortality in patients with T1DM than in patients without diabetes. Also, the results revealed that patients with T1DM have an approximately three-fold higher risk of ICU admission/mechanical ventilation and mortality when compared to patients with T2DM. The risk remained higher even after the results were adjusted for age, gender, and microvascular and macrovascular complications. To our knowledge, this is one of the most comprehensive studies published so far on T1DM patients with COVID-19.

There are several studies in the literature showing the relationship between COVID-19 disease and T2DM. Most of these studies reported that COVID-19 disease

\begin{tabular}{|c|c|c|c|c|c|c|c|c|c|c|}
\hline & \multicolumn{5}{|l|}{ T1DM vs. T2DM } & \multicolumn{5}{|c|}{ T1DM vs. nonDM } \\
\hline & & & & Odds Ratio (95\% Cl) & $\mathrm{p}$ value & & & & Odds Ratio (95\% Cl) & $\mathrm{p}$ value \\
\hline Hospitalization & & - & & $0.99(0.53-1.84)$ & 0.968 & & & & $1.74(1.21-2.50)$ & 0.003 \\
\hline ICU admission and/or intubation & & & - & $2.95(1.28-6.77)$ & 0.011 & & & — & $3.78(2.08-6.84)$ & $<0.001$ \\
\hline \multirow[t]{2}{*}{ Mortality } & & & - & $3.35(1.41-7.96)$ & 0.006 & & & & $6.44(3.27-12.67)$ & $<0.001$ \\
\hline & $\stackrel{4}{\text { Favors T2DM }}$ & 1 & $\underset{\text { Favors T1DM }}{\stackrel{0}{P}}$ & & & $\underset{\text { Favors nonDM }}{0 ?}$ & 1 & $\underset{\text { Favors } \mathrm{T10M}}{\stackrel{i 0}{\longrightarrow}}$ & & \\
\hline
\end{tabular}

Figure 2. The risks of COVID-19 outcomes in patients with type 1 diabetes mellitus (T1DM) relative to type 2 diabetes mellitus (T2DM) and non-diabetics; ICU - intensive care units 


\section{A}

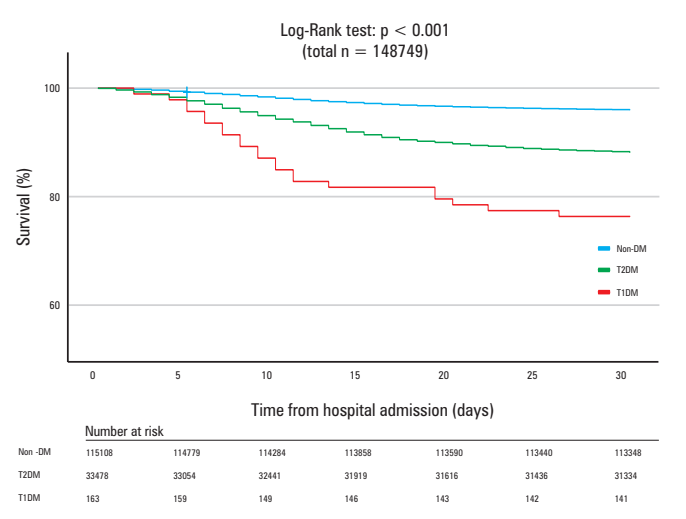

B

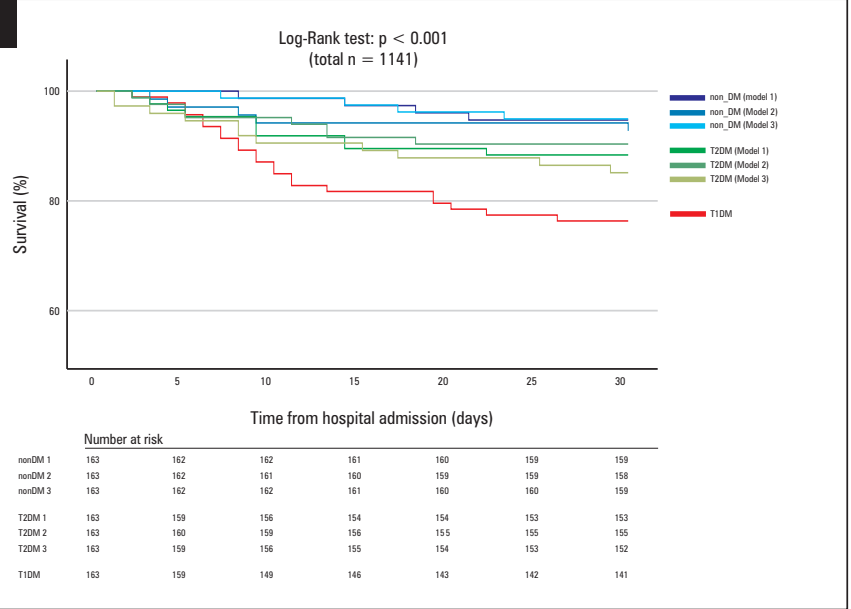

Figure 3. Kaplan-Meier survival curves showing crude (A) and propensity score matching (PSM) scenarios (B)

is quite common among T2DM patients, and generally with a more complicated course [5-7]. Very few studies have been published on COVID-19 in patients with T1DM; however, similar findings to T2DM have been reported [15-17, 19, 22, 23]. The low prevalence of T1DM in the general population, the relatively younger age of T1DM patients compared to T2DM patients, and the fact that the COVID-19 pandemic, at least at the beginning, affects older patients rather than younger ones are among the proposed reasons for the scarcity of data on T1DM $[24,25]$.

We observed a $16 \%$ overall mortality rate in COVID-19 patients with T1DM. Compared to the $1.8 \%$ mortality in the population without diabetes in the same dataset, such an increased risk deserves special attention because it seems to be one of the highest numbers reported studies so far. A whole-population study from England reported a 3.5\% death rate among T1DM patients $[16,20]$. The rate of mortality in the CORONADO study was $5.4 \%$ [23], while a recent UK study reported a $27 \%$ death rate in COVID-19 patients [22]. A multicentre and another small-scale study from the United States reported 9\% and 3\% death rates, respectively $[17,18]$. Higher mortality of patients with T1DM in our study may be explained by the increased burden of comorbidities. In a national registry from England, patients with T1DM who died due to COVID-19 had markedly higher rates of comorbidities including cardiovascular or renal comorbidities by $62.3 \%$, heart failure by $23.9 \%$, and stroke by $11.0 \%$ [20]. T1DM patients in our study had a more severe comorbidity burden, such as coronary artery disease, chronic kidney disease, and heart failure, by $39.9 \%, 54.4 \%$, and $23.3 \%$, respectively. Almost half of these patients had at least one microvascular complication.

In addition to the mortality outcome, the risk of hospitalization in the present study seems to be one of the highest among similar publications in the literature. Overall, six out of ten patients with T1DM in our study were hospitalized following COVID-19 diagnosis. Previous studies from different countries reported the hospitalization rates in patients with T1DM between $21.9 \%$ and $51 \%$. Also, almost one-third of our patients were admitted to the ICU, which was recorded as 5\% to $35 \%$ in other studies from different countries $[17,22,25$, $26]$. In the CORONADO study, $19.6 \%$ of patients with T1DM required mechanical ventilation [23]. Not surprisingly, the risk of a more severe COVID-19 course was higher in the T1DM population with a higher burden of significant comorbidities.

The present study compared T1DM and T2DM patients in terms of mortality and other prognostic factors using three different PSM models. In all models, the mortality rate was significantly higher in patients with T1DM, while there was no significant difference in the hospitalization, ICU admission, and intubation rates. Few studies so far have compared patients with T1DM and T2DM in terms of COVID-19 severity and mortality. One study reported fewer deaths in patients with T1DM compared to patients with T2DM (5.4\% vs. $10.6 \%)$, although the analysis was limited to 56 patients with T1DM [23]. In addition, there were significant differences in age and gender between the T1DM and T2DM groups. Another study from the UK reported that the odds of mortality from COVID-19 was 3.5 times higher in patients with T1DM and 2.0 times higher in those with T2DM relative to patients without diabetes [16]. In our three PSM models, the T1DM and T2DM groups were matched for age and gender, and the median age in both groups was 41 years. Although the data on the duration of diabetes duration was not available in our study, it is well-known that it is typically longer in individuals with T1DM than in those with T2DM of the same age because the onset of diabetes is 
much earlier in T1DM. Thus, one major reason for the increased mortality in patients with TIDM might be the longer duration of diabetes. Also, the median $\mathrm{HbA}_{1 \mathrm{c}}$ level in patients with T1DM in the present study was significantly higher than in the patients with T2DM. The risk of mortality from COVID-19 was reported to be higher at markedly increased $\mathrm{HbA}_{1 \mathrm{c}}$ levels in both T1DM and T2DM in a recent study from England [20]. Therefore, poor glycaemic control may also be involved in the mechanism of increased mortality in our study. For this reason, we conducted a further comparison of age, gender, $\mathrm{HbA}_{1 \mathrm{c}}$ levels, and microvascular and macrovascular complications between the matched groups of T1DM and T2DM. The results showed that patients with T1DM had higher mortality rates independently of $\mathrm{HbA}_{1 \mathrm{c}}$ levels and complications. These findings suggest that T1DM and T2DM are completely different diseases, and different immune dysfunctions in patients with T1DM may induce higher mortality rates in these patient groups.

Numerous studies have repeatedly identified older age as a significant factor in the course of COVID-19, not only in T2DM [27-29] but also in T1DM [16, 20, 25]. Our findings are consistent with the earlier findings that in COVID-19 patients with T1DM, age is a strong predictor of mortality and poor prognosis as well. Likewise, we identified lymphopaenia as a predictor of worse prognosis in the T1DM group, which is in line with the previous reports from others [7, 30,31]. In this regard, T1DM patients with older age and lymphopaenia should be treated more carefully during COVID-19.

Several limitations of the present study should be acknowledged. First, its observational design precludes establishing a causal relationship between the type of diabetes and outcomes. Second, all patients included in the study had a confirmed diagnosis of COVID-19 (PCR positive). The lack of symptomatic but unconfirmed COVID-19 cases or patients with false-negative COVID-19 PCR results may reduce the generalizability of our findings. Third, some data were unavailable in the dataset, such as duration of diabetes and insulin doses, which could be important factors to predict prognosis. And finally, the low number of patients with T1DM can be considered as a limitation. One major strength of this study is its population-based, nationwide design. Also, to our knowledge, this study is one of the most comprehensive reports of COVID-19 outcomes in adult patients with T1DM.

\section{Conclusion}

COVID-19 patients with T1DM have higher mortality rates than patients with T2DM and those without diabetes. The increased mortality risk in patients with
T1DM appears to be independent of age, gender, glycaemic control, and complications, suggesting that T1DM and T2DM have different pathophysiological mechanisms. Therefore, patients with T1DM seem to be particularly disadvantaged during the COVID-19 pandemic, suggesting some prioritization needs for prevention and care.

\section{Acknowledgements}

The authors gratefully acknowledge the Turkish healthcare professionals who worked hard for the management of COVID-19 sufferers during the outbreak and collected these data. We convey our deepest respect and gratitude to the memories of all our colleagues who lost their lives during the pandemic.

\section{Data availability}

The datasets generated during the current study are secured in a network and are not open to public sharing. The outputs can be copied following analysis but not the core patient registry. Practically, if requested in the future for any reason, additional information can be retrieved from the registry by contacting the corresponding author on reasonable request.

\section{Author contributions}

I.D., A.S., I.S., and I.T. were involved in the conceptualization and methodology of the study. N.A., O.C., M.C. and S.B. were responsible for the data download and verification. I.D., C.H., and I.T. performed the formal analysis and investigation. I.T., A.S., A.A., R.E., and I.S. critically reviewed and edited the first draft. All the authors were involved in the writing of the manuscript.

\section{Funding}

This research did not receive any specific grant from funding agencies in the public, commercial, or not-for-profit sectors.

\section{References}

1. Muller LM, Gorter KJ, Hak E, et al. Increased risk of common infections in patients with type 1 and type 2 diabetes mellitus. Clin Infect Dis. 2005; 41(3): 281-288, doi: 10.1086/431587, indexed in Pubmed: 16007521.

2. Allard R, Leclerc P, Tremblay C, et al. Diabetes and the severity of pandemic influenza A (H1N1) infection. Diabetes Care. 2010; 33(7): 1491-1493, doi: 10.2337/dc09-2215, indexed in Pubmed: 20587722.

3. Sanyaolu A, Okorie C, Marinkovic A, et al. Comorbidity and its Impact on Patients with COVID-19. SN Compr Clin Med. 2020 [Epub ahead of print]: 1-8, doi: 10.1007/s42399-020-00363-4, indexed in Pubmed: 32838147.

4. Fathi M, Vakili K, Sayehmiri F, et al. The prognostic value of comorbidity for the severity of COVID-19: A systematic review and meta-analysis study. PLoS One. 2021; 16(2): e0246190, doi: 10.1371/journal. pone.0246190, indexed in Pubmed: 33592019.

5. Sun Y, Guan X, Jia L, et al. Independent and combined effects of hypertension and diabetes on clinical outcomes in patients with COVID-19: A retrospective cohort study of Huoshen Mountain Hospital and Guanggu Fangcang Shelter Hospital. J Clin Hypertens (Greenwich). 2021; 23(2): 218-231, doi: 10.1111/jch.14146, indexed in Pubmed: 33369066. 
6. Dennis JM, Mateen BA, Sonabend R, et al. Type 2 Diabetes and COVID-19-Related Mortality in the Critical Care Setting: A National Cohort Study in England, March-July 2020. Diabetes Care. 2021; 44(1): 50-57, doi: 10.2337/dc20-1444, indexed in Pubmed: 33097559.

7. Sonmez A, Demirci I, Haymana C, et al. Clinical characteristics and outcomes of COVID-19 in patients with type 2 diabetes in Turkey: A nationwide study (TurCoviDia). J Diabetes. 2021; 13(7): 585-595, doi: 10.1111/1753-0407.13171, indexed in Pubmed: 33655669.

8. Carrasco-Sánchez FJ, López-Carmona MåD, Martínez-Marcos FJ, et al. SEMI-COVID-19 Network. Admission hyperglycaemia as a predictor of mortality in patients hospitalized with COVID-19 regardless of diabetes status: data from the Spanish SEMI-COVID-19 Registry. Ann Med. 2021; 53(1): 103-116, doi: 10.1080/07853890.2020.1836566, indexed in Pubmed: 33063540.

9. Wang D, Hu Bo, Hu C, et al. Clinical Characteristics of 138 Hospitalized Patients With 2019 Novel Coronavirus-Infected Pneumonia in Wuhan, China. JAMA. 2020; 323(11): 1061-1069, doi: 10.1001/jama.2020.1585, indexed in Pubmed: 32031570.

10. Alguwaihes AM, Al-Sofiani ME, Megdad M, et al. Diabetes and Covid-19 among hospitalized patients in Saudi Arabia: a single-centre retrospective study. Cardiovasc Diabetol. 2020; 19(1): 205, doi: 10.1186/s12933-020-01184-4, indexed in Pubmed: 33278893.

11. Huang I, Lim MA, Pranata R. Diabetes mellitus is associated with increased mortality and severity of disease in COVID-19 pneumonia - A systematic review, meta-analysis, and meta-regression. Diabetes Metab Syndr. 2020; 14(4): 395-403, doi: 10.1016/j.dsx.2020.04.018, indexed in Pubmed: 32334395.

12. Cariou B, Pichelin M, Goronflot T, et al. CORONADO investigators, CORONADO investigators, CORONADO investigators, CORONADO investigators. Phenotypic characteristics and prognosis of inpatients with COVID-19 and diabetes: the CORONADO study. Diabetologia. 2020; 63(8): 1500-1515, doi: 10.1007/s00125-020-05180-x, indexed in Pubmed: 32472191.

13. Agarwal S, Schechter C, Southern W, et al. Preadmission Diabetes-Specific Risk Factors for Mortality in Hospitalized Patients With Diabetes and Coronavirus Disease 2019. Diabetes Care. 2020; 43(10): 2339-2344, doi: 10.2337/dc20-1543, indexed in Pubmed: 32769128.

14. Zhu L, She ZG, Cheng Xu, et al. Association of Blood Glucose Contro and Outcomes in Patients with COVID-19 and Pre-existing Type 2 Diabetes. Cell Metab. 2020; 31(6): 1068-1077.e3, doi: 10.1016/j.cmet.2020.04.021, indexed in Pubmed: 32369736.

15. Fisher L, Polonsky W, Asuni A, et al. The early impact of the COVID-19 pandemic on adults with type 1 or type 2 diabetes: A national cohor study. J Diabetes Complications. 2020; 34(12): 107748, doi: 10.1016/j. jdiacomp.2020.107748, indexed in Pubmed: 33059981.

16. Barron E, Bakhai C, Kar P, et al. Associations of type 1 and type 2 diabetes with COVID-19-related mortality in England: a whole-population study. Lancet Diabetes Endocrinol. 2020; 8(10): 813-822, doi: 10.1016/S2213-8587(20)30272-2, indexed in Pubmed: 32798472

17. O'Malley G, Ebekozien O, Desimone M, et al. COVID-19 Hospitalization in Adults with Type 1 Diabetes: Results from the T1D Exchange Multicenter Surveillance Study. J Clin Endocrinol Metab. 2021; 106(2): e936-e942, doi: 10.1210/clinem/dgaa825, indexed in Pubmed: 33165563.

18. Ebekozien OA, Noor N, Gallagher MP, et al. Type 1 Diabetes and CO VID-19: Preliminary Findings From a Multicenter Surveillance Study in the U.S. Diabetes Care. 2020; 43(8): e83-e85, doi: 10.2337/dc20-1088, indexed in Pubmed: 32503837.

19. Vamvini M, Lioutas VA, Middelbeek RJW. Characteristics and Diabetes Control in Adults With Type 1 Diabetes Admitted With COVID-19 Infection. Diabetes Care. 2020; 43(10): e120-e122, doi: 10.2337/dc20-1540, indexed in Pubmed: 32769127.

20. Holman N, Knighton P, Kar P, et al. Risk factors for COVID-19-related mortality in people with type 1 and type 2 diabetes in England: a population-based cohort study. Lancet Diabetes Endocrinol. 2020; 8(10): 823-833, doi: 10.1016/S2213-8587(20)30271-0, indexed in Pubmed: 32798471.

21. Levey AS, Stevens LA, Schmid CH, et al. CKD-EPI (Chronic Kidney Disease Epidemiology Collaboration). A new equation to estimate glomerular filtration rate. Ann Intern Med. 2009; 150(9): 604-612, doi: 1 0.7326/0003-4819-150-9-200905050-00006, indexed in Pubmed: 19414839.

22. Ruan Y, Ryder REJ, De P, et al. ABCD Covid-19 audit group. A UK nationwide study of people with type 1 diabetes admitted to hospital with COVID-19 infection. Diabetologia. 2021; 64(8): 1717-1724, doi: 10.1007/s00125-021-05463-x, indexed in Pubmed: 33966090.

23. Wargny M, Gourdy P, Ludwig L, et al. CORONADO investigators. Type 1 Diabetes in People Hospitalized for COVID-19: New Insights From the CORONADO Study. Diabetes Care. 2020; 43(11): e174-e177, doi: 10.2337/dc20-1217, indexed in Pubmed: 32847826.

24. Pitocco D, Tartaglione L, Viti L, et al. Lack of type 1 diabetes involvemen in SARS-COV-2 population: Only a particular coincidence? Diabetes Res Clin Pract. 2020; 164: 108220, doi: 10.1016/j.diabres.2020.108220, indexed in Pubmed: 32442557.

25. Gregory JM, Slaughter JC, Duffus SH, et al. COVID-19 Severity Is Tripled in the Diabetes Community: A Prospective Analysis of the Pandemic's Impact in Type 1 and Type 2 Diabetes. Diabetes Care. 2021; 44(2): 526-532, doi: 10.2337/dc20-2260, indexed in Pubmed: 33268335.

26. Al Hayek AA, Robert AA, Alotaibi ZK, et al. Clinical characteristics of hospitalized and home isolated COVID-19 patients with type 1 diabetes. Diabetes Metab Syndr. 2020; 14(6): 1841-1845, doi: 10.1016/j. dsx.2020.09.013, indexed in Pubmed: 32971511.

27. Wang L, He W, Yu X, et al. Coronavirus disease 2019 in elderly patients: Characteristics and prognostic factors based on 4-week follow-up. J Infect. 2020; 80(6): 639-645, doi: 10.1016/j.jinf.2020.03.019, indexed in Pubmed: 32240670

28. Ho FK, Petermann-Rocha F, Gray SR, et al. Is older age associated with COVID-19 mortality in the absence of other risk factors? General population cohort study of 470,034 participants. PLoS One. 2020; 15(11): e0241824, doi: 10.1371/journal.pone.0241824, indexed in Pubmed: 33152008.

29. Shahid Z, Kalayanamitra R, McClafferty B, et al. COVID-19 and Older Adults: What We Know. J Am Geriatr Soc. 2020; 68(5): 926-929, doi: 10.1111/jgs.16472, indexed in Pubmed: 32255507.

30. Huang I, Pranata R. Lymphopenia in severe coronavirus disease-2019 (COVID-19): systematic review and meta-analysis. J Intensive Care. 2020; 8: 36, doi: 10.1186/s40560-020-00453-4, indexed in Pubmed: 32483488.

31. Muñoz-Rodríguez JR, Gómez-Romero FJ, Pérez-Ortiz JM, et al. COVID-19 SESCAM Network. Characteristics and Risk Factors Associated With Mortality in a Multicenter Spanish Cohort of Patients With COVID-19 Pneumonia. Arch Bronconeumol. 2021; 57: 34-41, doi: 10.1016/j. arbres.2021.02.021, indexed in Pubmed: 34629641. 